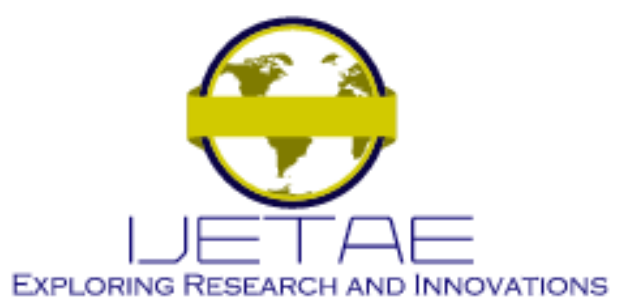

International Journal of Emerging Technology and Advanced Engineering

Website: www.ijetae.com (E-ISSN 2250-2459, Scopus Indexed, ISO 9001:2008 Certified Journal, Volume 11, Issue 10, October 2021)

Manuscript Received: 02 September 2021, Received in Revised form: 03 October 2021, Accepted: 10 October 2021

DOI: 10.46338/ijetae1021_04

\title{
Research and Development of Gearbox Load Spectrum Database under Variable Working Conditions
}

\author{
Changlun $\mathrm{Gao}^{1}$, Junliang $\mathrm{Huo}^{2}$, Zhantong $\mathrm{Li}^{3}$, Beihuo $\mathrm{Yao}^{4}$, Zhaokai $\mathrm{He}^{5}$, Fangren Zhang ${ }^{6}$, Shunli Sun ${ }^{7}$, Weibin $\mathrm{Wu}^{8}$ \\ 1,2,3,4,5,6,7 Collage of Engineering, South China Agricultural University Guangzhou 510642, China
}

\begin{abstract}
Gearbox load spectrum is the data basis for gearbox fatigue life prediction and strength verification. As the amount of load spectrum data increases exponentially and the demand for sharing increases, it is necessary to introduce database technology to store and manage load spectrum data. In this paper, the collection and processing of load data and the compilation of load spectrum are introduced in turns firstly. Then it sums the development and research status of database technology and the technical route of combining load spectrum database up secondly. Moreover, the current status of load spectrum database and the design steps of load spectrum database are summarized. Finally, the development trend of load spectrum database research is generalized and the prospect is put forward.
\end{abstract}

Keywords-Load spectrum; database technology; development trend; gear box

\section{RESEARCH BACKGROUND AND IMPORTANCE}

Gearbox is a functional component that passes motion in automobile, construction machinery and general machinery products. It is gearbox which plays a key role in the powertrain of new energy vehicles particularly in its design that used to change the speed and torque of machinery and also a complex of shaft, bearing, gear, box and other parts. Figure 1 is the gearbox of a new energy vehicle. The failure of gearbox accounts for more than $50 \%$ of the failure of the whole system in the actual operation of the project ${ }^{[1]}$. Therefore, it is important to put the research on the fatigue failure of gearbox which focuses on the analysis of the fatigue life of gearbox. At present, the research on the fatigue life of gearbox mostly focuses on three aspects: load spectrum acquisition, fatigue test and life analysis method. All of the above are inseparable from the support of load spectrum data ${ }^{[2]}$.

Automobile load spectrum is the main object of automobile reliability research. At the same time, it is often used as a reference to estimate the fatigue life of parts. Load spectrum has become the data basis for new energy vehicle design, performance, power train fatigue life prediction and strength verification.
Load spectrum simulation and test loading can not only save cost, but also improve test efficiency and reduce labour intensity. It is of great significance for the research, testing and performance improvement of core components such as gearbox of new energy vehicles ${ }^{[3]}$.

In the gearbox project, multi-conditions load spectrum from various regions need to be collected and processed. It can not only characterize the load characteristics of the corresponding regional road conditions, but also reflect the impact of local driving conditions on the fatigue life of the reducer gear in the reducer bench fatigue test preferably. In the face of such a large amount of complex load spectrum data, the traditional data storage methods excel and MATLAB obviously can't meet the functions of data analysis, storage, query and export. Therefore, it is necessary to introduce database technology to store the massive original load data and processed regional multi condition program load spectrum data in the project of Gearbox load spectrum, in order to facilitate query according to regional conditions. The database interface is shown in Figure 2. The establishment of load spectrum database not only provides more targeted data for the development of reducer gear, but also provides data support for the future study of gearbox fatigue life and strength verification.

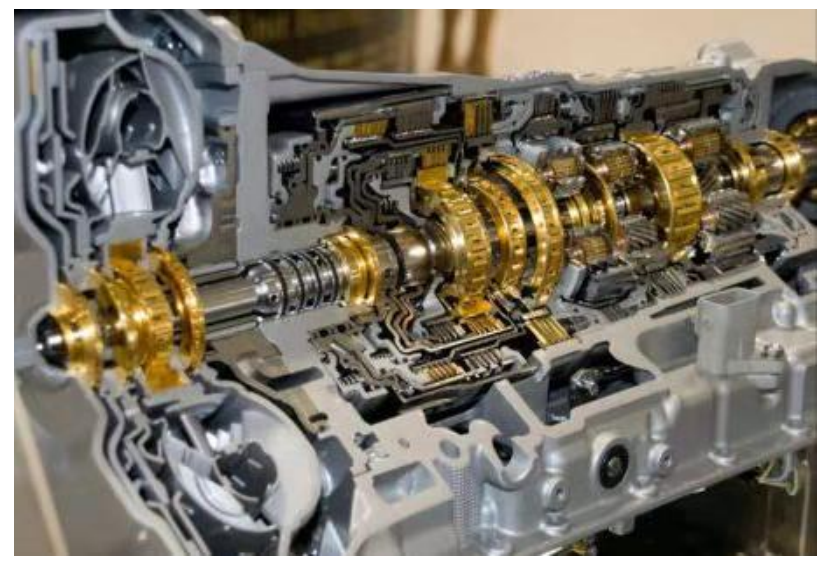

Fig 1 Figure of gearbox of new energy vehicle 


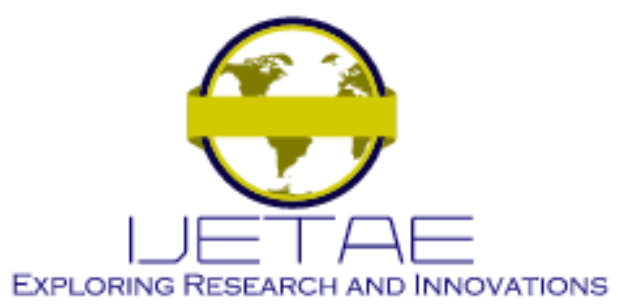

International Journal of Emerging Technology and Advanced Engineering

Website: www.ijetae.com (E-ISSN 2250-2459, Scopus Indexed, ISO 9001:2008 Certified Journal, Volume 11, Issue 10, October 2021)

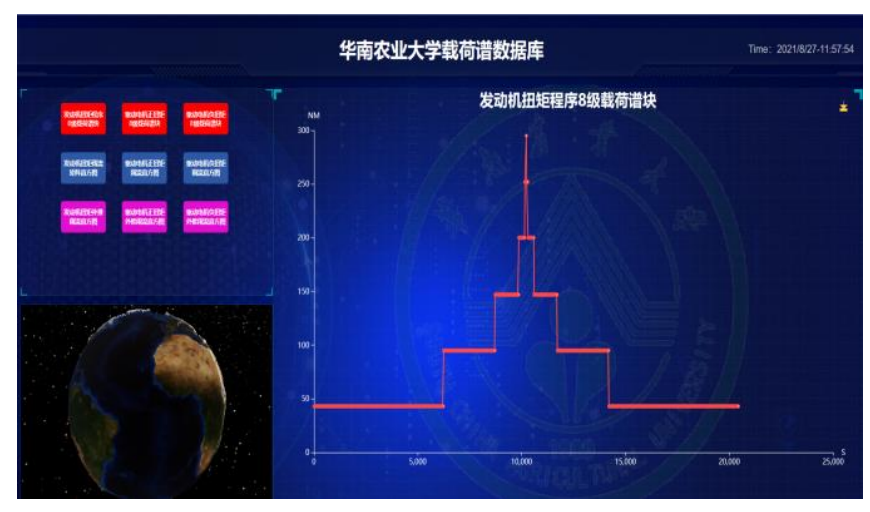

Fig 2 Figure of Database of gearbox load spectrum

\section{COMPILING Method Of LOAD SPECTRUM}

The compilation of load spectrum is mainly divided into three steps: the collection and processing of load data and the compilation of load spectrum.

\section{A. Processing of load data}

The obtained data can't accurately reflect the loading situation of the gearbox in the whole life stage in view of the complexity of the test environment, the diversity of test conditions, the influence of test time and various accidental factors. It needs to be analysed and reprocessed as the date is collected by the gearbox will be subject to various disturbances which may lead not accurately reflect the loading situation of the gearbox in the whole life stage. The analysed methods mainly include the stationary randomness analysis, correlation analysis, statistical calculation, selection of the best sample, elimination of data outliers, centralized processing, resampling and filtering of data, etc, and then the load spectrum is prepared ${ }^{[4]}$. Two aspects of singular value elimination and invalid amplitude filtering are emphatically considered in view of the load data processing of automobile gearbox.

\section{1) Singularity removal of load data}

The sudden changes of equipment current or the influence of external environment can cause signal acquisition to produce singular values, which are characterized by few sampling points, strong randomness or large values.
The load signal is affected by temperature change and other factors, and the data drift phenomenon is more common, so it is necessary to remove the singularity of the collected load data. Taking the torque load of a section of drive motor as an example, the upper figure of Figure 3 shows before processing and the lower figure shows after processing. It can be seen that the corresponding abnormal value has been removed.

There are three common methods to remove singular points: gradient threshold detection method, standard variance detection method and amplitude threshold method. The amplitude threshold detection method can't effectively distinguish normal signals from small amplitude abnormal signals; Gradient threshold detection method can't effectively distinguish abnormal value from normal signal with large variation amplitude; The standard variance detection method needs to set the variance threshold according to the understanding and experience of the data ${ }^{[5]}$. The above three methods have their own advantages and disadvantages in actual data processing, and need to be combined for comprehensive debugging to meet the actual needs.

In regard to the removal of singular points, Wang etc. applied the classification analysis technology to the noise reduction effect evaluation of load signals of transmission parts of engineering vehicles, and proposed that we should mainly pay attention to the relationship between probability density function and load distribution characteristics, cumulative distribution function and frequency spectrum ${ }^{[6]}$; Xiao etc. obtained the load time history of the crane under different working conditions by using the finite element dynamic simulation method in the field test. This method has the advantages of ensuring the accuracy of load data and reducing the amount of data as much as possible ${ }^{[7]}$; Zhang etc. combined the amplitude threshold detection method, gradient threshold detection method and variance detection method to remove the abnormal peak points in the signal, and the real signal reflecting the load change law of the transmission system can be obtained ${ }^{[8]}$; Mou etc. proposed a blink artifact removal method based on wavelet singularity detection and threshold denoising. This method has good applicability for signals with different frequencies ${ }^{[9]}$. 


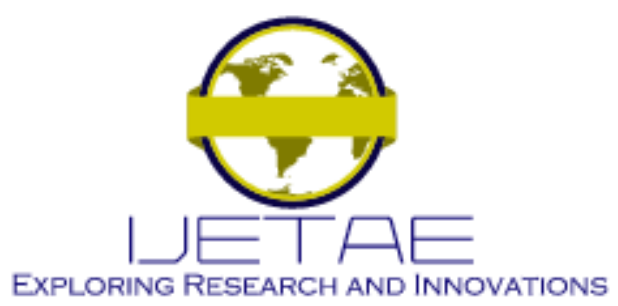

International Journal of Emerging Technology and Advanced Engineering

Website: www.ijetae.com (E-ISSN 2250-2459, Scopus Indexed, ISO 9001:2008 Certified Journal, Volume 11, Issue 10, October 2021)
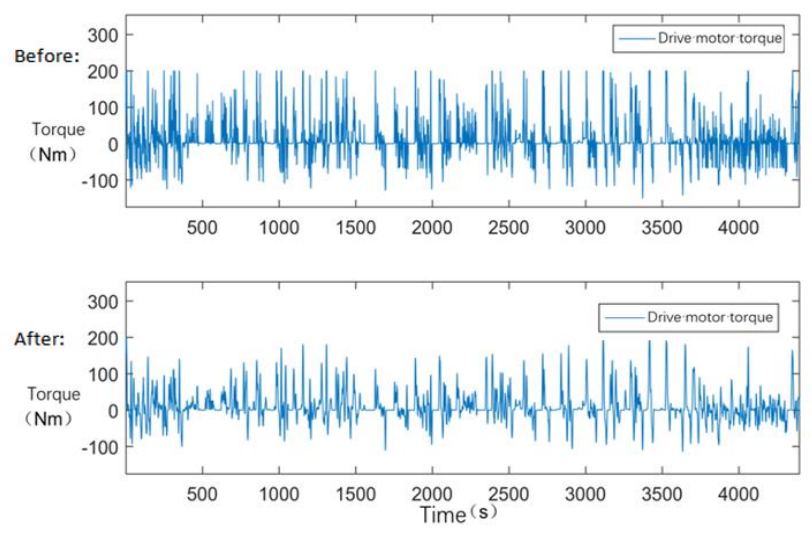

Fig 3 Comparison diagram of before and after singular point removal

\section{2) Invalid amplitude filtering}

Invalid amplitude refers to a small number of cycles caused by some secondary loads that cannot constitute fatigue damage. A measured random load spectrum is often formed by many factors. In addition to the main working load, it is often affected by some secondary loads. These loads are represented by second-order waves, third-order waves and even some high-order small cycles. These small cycles that cannot constitute fatigue damage are often abandoned, to reduce the cost of fatigue test and avoid unnecessary waste of test cycle. As for the trade-off benchmark of invalid amplitude, 5\% - 10\% of the range of random load history (maximum stress - minimum stress) is generally taken ${ }^{[10]}$. Taking the torque of a driving motor in the project of Gearbox load spectrum, the original load time history is $4600 \mathrm{~s}$, as shown in the upper figure of Figure 4. After removing the invalid amplitude, the load time history is only about 1500 s, as shown in the lower figure of Figure 3.

There are many methods to determine the removal standard of small load, and its purpose is to reasonably remove the load cycle below a certain limit. Xu etc, proposed a method to remove small cycles according to the fatigue strength limit standard, and its rationality was verified by calculation ${ }^{[11]}$; Guo etc, discovered through research that in order to ensure the accuracy of fatigue damage, $5 \%$ of the range in the load time history can be used as the abandonment standard of invalid load ${ }^{[12]}$.
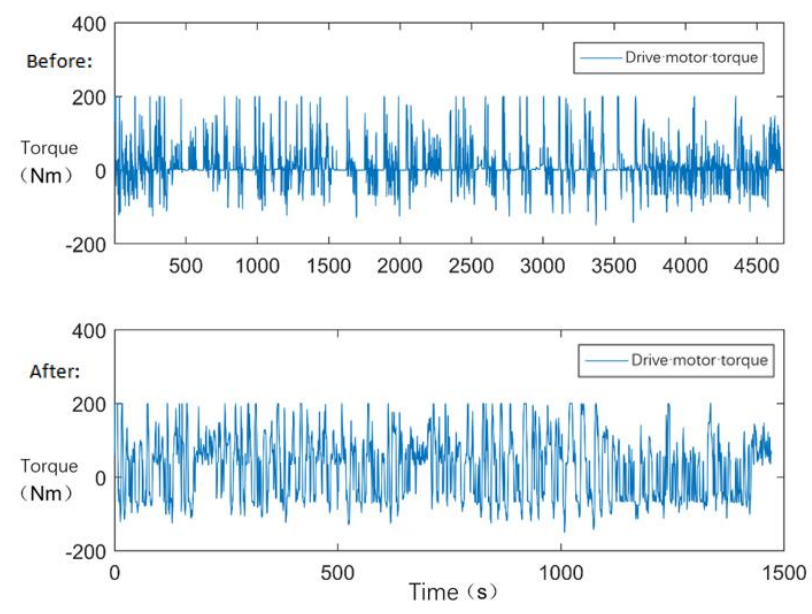

Fig 4 Comparison diagram of before and after invalid amplitude filtering

\section{B. Research status of load spectrum counting method}

When sufficient random stress data are obtained, these data need to be converted into load spectrum that can be used for fatigue, durability, damage volume analysis and test. Therefore, statistical analysis should be carried out for various fatigue load environmental parameters and fatigue load itself. At present, the commonly used statistical methods of random stress mainly include power spectrum method and cycle counting method.

\section{1) Method of cycle counting}

Cycle counting is to simplify the complex load time history into a load time history expressed in full cycle and half cycle, which is easy to control. This simplified process is called counting method. Based on the fatigue damage theory, the load information is obtained by counting the load frequency of the same group in the complex random load.

This method is more in line with the needs of engineering research which has the advantages of simple principle and strong operability. Although there are problems such as loss of loading sequence, the loading sequence generally has little effect on fatigue life ${ }^{[13]}$.

In the study of fatigue problems, the counting method can be divided into single parameter counting method and double parameter counting method. 


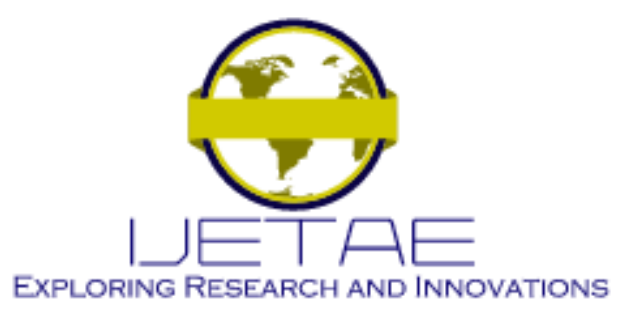

International Journal of Emerging Technology and Advanced Engineering

Website: www.ijetae.com (E-ISSN 2250-2459, Scopus Indexed, ISO 9001:2008 Certified Journal, Volume 11, Issue 10, October 2021)

Single parameter counting methods include peak counting method, step through counting method and cross mean peak counting method. The double parameter counting method can record all the information of load, which is a better counting method in engineering. Double parameter counting methods include rain flow counting method, vibration range mean counting method, etc ${ }^{[14]}$. At present, the rain flow counting is the most widely used method in the project. Rain flow counting method can not only truly reflect the fatigue process of materials, but also have the characteristics of programmability. It can be used to compile program loading spectrum. Therefore, it has been studied and used by many researchers. The counting method of rain flow counting is like raindrops flowing down the roof. Its principle is shown in Figure 5.

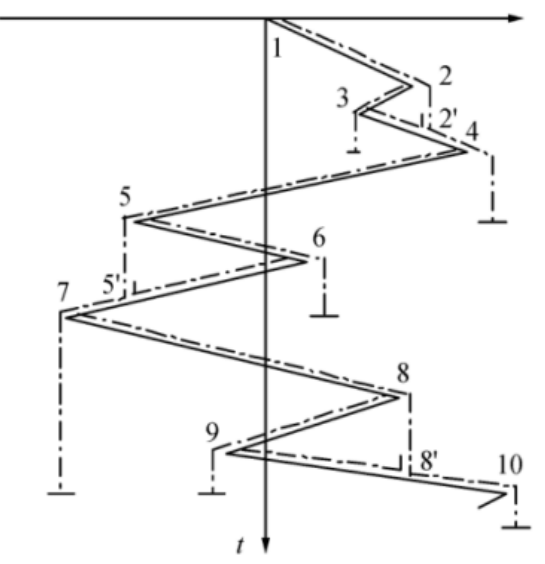

Fig 5 Schematic diagram of rain flow counting method ${ }^{[15]}$

In terms of research and optimization, Yan etc. improved the previous rain flow counting method to make the optimized rain flow counting method have the ability of real-time operation ${ }^{[16]}$; Zhang etc. proposed the representative spectrum method by studying the rain flow counting method, which can truly reflect the loading sequence of the load ${ }^{[17]}$; Jiang etc. made the rain flow counting method capable of counting under intermittent conditions $^{[18]}$.

In terms of use, Gao etc. took a certain type of gear bearing periodic load for a long time as the research object, analysed the load condition of gear teeth in gear meshing.
They determined the gear dynamic load spectrum as sample extraction, realized the gear dynamic load spectrum counting based on rain flow counting recursive algorithm, and further determined the gear program load spectrum ${ }^{[19]}$; Beak etc. used rain flow cycle counting to evaluate the cumulative damage of end beam under operating load ${ }^{[20]}$; Zheng etc. used an improved three peak valley rain flow counting method to discuss the fatigue damage assessment method of hull structure in view of the vulnerability of hull structure to fatigue and other damage during ship operation $^{[21]}$.

\section{2) Method of power spectrum}

The power spectrum method decomposes the random stress into the sum of ordered multiple frequency band processes by using Fourier transform (FFT), and retains the data information required for statistical analysis in the load time history ${ }^{[22]}$. The advantage of power spectrum method is that it can retain all the information of load, but this method has poor operability and narrow adaptability, and it is too complex to calculate the random load distribution.

There are two widely used power spectrum methods: parametric estimation method and nonparametric estimation method. The parameter estimation method is to solve the minimum parameters of the given function. This method mainly includes Burg's entropy function method and autocorrelation method; Yu etc. used the new Weibull distribution parameter estimation method to analyse the life reliability of the engine ${ }^{[23]}$.

The nonparametric estimation method does not make any assumptions about the model itself and the generation process of data, such as periodic graph method, improved periodic method and Welch method. For the periodogram method based on discrete Fourier transform in the power spectrum estimation method, $\mathrm{Fu}$ etc, proposed the piecewise average periodogram method in the improved nonparametric method ${ }^{[24]}$.

\section{Compilation of load spectrum}

In the process of compiling load spectrum, there are a lot of compiling methods to choose. The commonly used compiling methods of load spectrum include fluctuation center method, variable mean method and equal life relationship transformation method. 


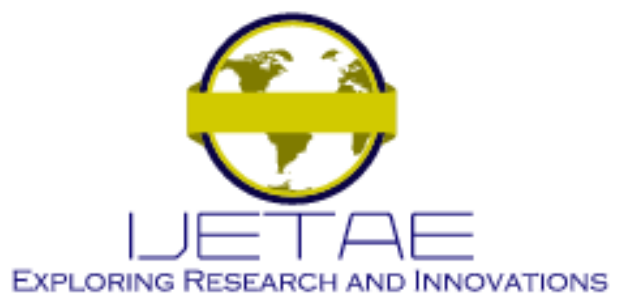

International Journal of Emerging Technology and Advanced Engineering

Website: www.ijetae.com (E-ISSN 2250-2459, Scopus Indexed, ISO 9001:2008 Certified Journal, Volume 11, Issue 10, October 2021)

\section{1) Wave center method}

It is generally believed that the amplitude change of random load time history has a great impact on the fatigue life of metal structure parts. If the mean value and amplitude of load in the load history are regarded as binary random variables, the analysis process will become very complex. The wave center method is to simplify the obtained mean and amplitude of fatigue load into onedimensional random variables for analysis and calculation, that is, dimensionality reduction. In the process of compiling the load spectrum, the overall average value of the mean value of all load cycles takes the fluctuation center as the static part of the load cycle and the amplitude as the dynamic part, and the amplitude is superimposed on the fluctuation center. In this way, the obtained load data can be well used to compile the load spectrum without considering the distribution of the mean value.

When the wave center is obtained by the wave center method, the distribution of load amplitude needs to be analysed and considered. The rain flow counting method is used to count and analyse the data, and the data are grouped according to the amplitude frequency of the data. The load amplitude in each group of data is expressed by the group median, and then the frequency occurrence times are accumulated to obtain the excess cumulative frequency.

\section{2) Variable average method}

The wave center method is usually applicable to the load time history with symmetrical characteristics. For the asymmetric load time history, the wave center method is used to simplify the operation. The wave center method will replace the average value in all load cycles with the unified overall average value, resulting in serious distortion. The results obtained will be very different from the actual situation. Therefore, for the asymmetric load time history, the variable average method is recommended. The variable average method does not need to use the unified overall average value when making statistics. It divides the loads with the same amplitude into a group to obtain the "group average value" of each group, so as to obtain the regular curve of the group average value of the load mean value varying with the amplitude, and replace the average value of the overall mean value. The new load curve can be obtained by superimposing the amplitude with the average value of the group mean.

\section{3) Equal life relationship transformation method}

The equal life relationship transformation method is based on Gerber, Goodman and other calculation methods to equivalently transform the load mean in the load time history into the amplitude curve, so as to obtain the onedimensional load cycle similar to that obtained by counting and analysis by single parameter counting method, and then carry out statistical calculation and analysis.

\section{RESEARCH StATUS Of LOAD SPECTRUM DATABASE}

\section{A. Overview of database technology}

Database technology is an effective technology of data management. Database is an important part of computer technology. It is not only applied to transaction processing, but also can be further applied to the fields of information retrieval, artificial intelligence, expert system, computeraided design and so on. Database technology came into being in the late 1960s and early 1970s. Its development has experienced file system stage, manual management stage, database system stage and advanced database system stage. Database technology continues to develop and improve, and the application field continues to expand.

\section{1) Data model}

Data model is not only the abstraction of real-world data characteristics, but also the core and foundation of database system. Data model is divided into conceptual model, logical model and physical model. Generally speaking, a data model is a set of strictly defined concepts. These concepts accurately describe the static characteristics, dynamic characteristics and integrity constraints of the system. Therefore, data model usually consists of three parts: data structure, data operation and data integrity.

The most common data models in the field of database theory mainly include hierarchical model, mesh model and relational model. Among them, relational model is the most important data model, and it is also the most widely used data model in the design of load spectrum database.

The relational model is based on strict mathematical concepts. It has single concept, clear data structure, higher data independence and better security. The above advantages make the relational model deeply loved by users, and has been rapidly developed and widely used. 


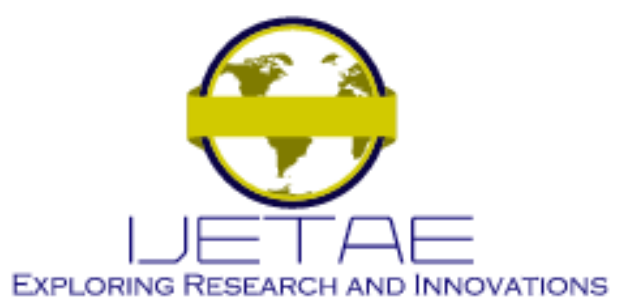

International Journal of Emerging Technology and Advanced Engineering Website: www.ijetae.com (E-ISSN 2250-2459, Scopus Indexed, ISO 9001:2008 Certified Journal, Volume 11, Issue 10, October 2021)

\section{2) Database System}

Database system is a system composed of database and its management software. It is generally composed of database, hardware, software and personnel. The most important personnel are developer and database administrator. Developer is responsible for the development of the database. The database administrator is responsible for defining the security requirements and integrity constraints of the database, monitoring the use and operation of the database, and improving the performance, reorganization and reconstruction of the database to improve the performance of the system ${ }^{[25]}$. The structure of the database system is shown in Figure 6.

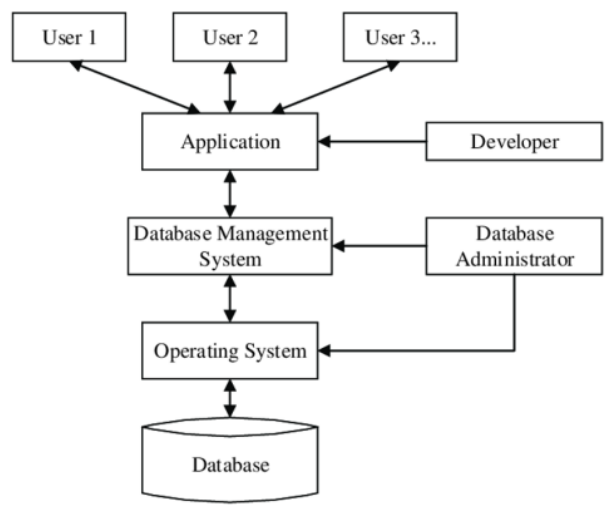

Fig 6 Diagram of database system structure

\section{B. Research status of load spectrum database}

Data, application requirements and computer hardware technology are the three main driving forces or three important factors to promote the development of database. At present, the application of database technology is mainly manifested in the integrated development with various technologies. The load spectrum database is one of the products of the integrated development. The load spectrum database resources have the following characteristics:

(1) The amount of data is huge. Taking the earlier aircraft load spectrum as an example, the amount of data in 2007 is roughly: more than 500 parameters and more than 100 billion sample measured data. Moreover, with the improvement of test technology and recording ability, the number of measured parameters and data sampling rate of aircraft load spectrum measured data are also higher and higher, and the amount of data is also increasing exponentially ${ }^{[26]}$.
(2) For the measured data of load spectrum of different research objects, the number of parameters, sampling rate and data type are different, which is difficult to store in a unified structured data table ${ }^{[27]}$.

(3) Different types of measured data structures, data processing methods and load spectrum preparation methods are also different, and the methods and results of data processing and load spectrum preparation need to be recorded ${ }^{[28]}$.

The load spectrum database generally contains a large number of load spectrum information of different machines under different working conditions, as well as user information, material information, life calculation results and other information required by the platform. Data storage involves many data and types. Therefore, the relational database with higher data independence, better security and better abstraction of reality should be selected.

\section{Selection of load spectrum database}

At present, the mainstream relational databases on the market include SQL Server and Oracle, MySQL, DB2, Access, PostgreSQL, etc ${ }^{[29]}$.

In combination with the above, if there is a huge amount of data in the load spectrum database and there are sharing and parallel requirements, you can choose Oracle or DB2, a large database management system with strong performance, the highest security and extremely stable. As distributed databases, they have large volume, high cost, cross platform use, strong scalability, support concurrent operation, strong real-time performance, and have database recovery function. Among them, Oracle has the highest performance, while DB2 is relatively simple to operate.

For the load database built by a company and a unit, the amount of data is relatively large, and only the data needs to be shared in the company's internal LAN. SQL server has comprehensive functions and high efficiency. It can be used as a database platform for medium-sized enterprises or units. SQL Server database can be used. SQL server is a good choice for various enterprise information management systems developed on Windows platform, whether client / server architecture or browser / server architecture.

In the case of individual, the demand of establishing personal load spectrum database with low cost should be properly considered. Free databases such as SQLite, PostgreSQL and MySQL can be considered, which has high security and small volume. 


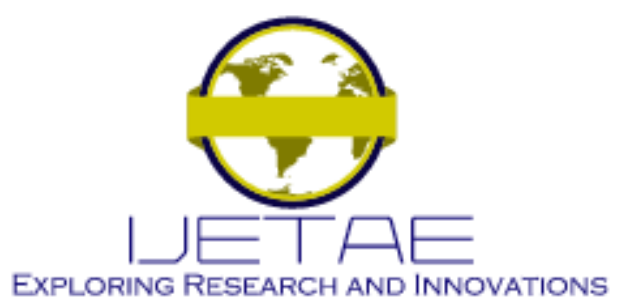

International Journal of Emerging Technology and Advanced Engineering

Website: www.ijetae.com (E-ISSN 2250-2459, Scopus Indexed, ISO 9001:2008 Certified Journal, Volume 11, Issue 10, October 2021)

However, due to the large amount of load spectrum data, the performance of the above database is poor in the case of a large amount of data.

\section{Architecture of common load spectrum database system}

Common system structures include Client / Server architecture and Browser / Server architecture. Other system architectures are evolved and combined from the above two architectures, so the above two architectures are mainly introduced.

\section{1) Client server architecture of database}

The client server architecture of database is a typical two-tier architecture, which is composed of client and service. Its basic architecture is shown in Figure 7.

The user's program is mainly on the client, which is responsible for sending request, response request, view display and other human-computer interaction. The server mainly provides data management, data sharing, data and system maintenance and concurrency control. The user completes the specific business at the client, sends the request to the server, the server then requests the database server, and finally responds to the requests of multiple clients by means of distributed processing.

C / S mode is relatively safe and reliable, easy to develop, but poor portability. In the face of users of different operating systems, we must spend a lot of time and energy redeveloping the client of the corresponding system.

Yan etc. developed the median random load spectrum data processing expert system in $\mathrm{C} / \mathrm{S}$ mode. The client adopts mixed programming of $\mathrm{C}$ language and assembly language. The database is deployed on the server and uniformly called by the database management system ${ }^{[30]}$; The environmental information database model proposed by Tian etc. Is also based on the client / server model, and then combined with professional knowledge such as material science and corrosion fatigue to build an aircraft calendar life calculation and evaluation system ${ }^{[31]}$; Shen etc. built the tower crane residual life evaluation system, and divided the C / S mode into the representation part on the client side and the application logic part and data access part on the server side. The data access part corresponds to the database server. The server is only deployed in the company's LAN, which greatly improves the communication efficiency ${ }^{[32]}$.

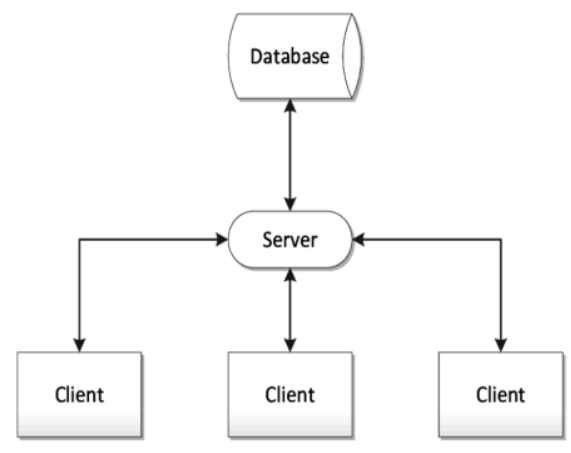

Fig 7 Mode of C/S architecture

\section{2) Browser and server architecture of database}

B / S architecture, that is, browser / server architecture. It consists of logically separated presentation layer, business layer and data layer. The presentation layer, that is, the browser provides data to customers, the business layer implements business and data rules, and the data layer, that is, the database, defines data access standards and stores data. Its basic architecture is shown in Figure 8.

The B / S system unifies the client without special installation; It concentrates the core part of the system function realization on the server, which simplifies the development, maintenance and use of the system. The database can be installed on the server, and then the browser transmits information through the web server and database server through HTTP request response.

B / S mode has the characteristics of strong portability, cross platform and flexibility. B / S multiple considerations on security and access speed are based on the need for more optimization. However, with the development of JavaScript technology and Ajax technology, as well as the security protection measures of mainstream browsers, the security of data has been greatly guaranteed.

The fatigue life analysis system based on damage tolerance constructed by $\mathrm{Xu}$ etc. who considered the useroriented personnel are complex, the platform needs to be simple and easy, and meets the needs of cross regional communication of users $^{[41]}$. So, they chose B / S structure for system development; The network-based multiaxial strain fatigue analysis platform built by Liu etc. who adopted B / S mode as well. 


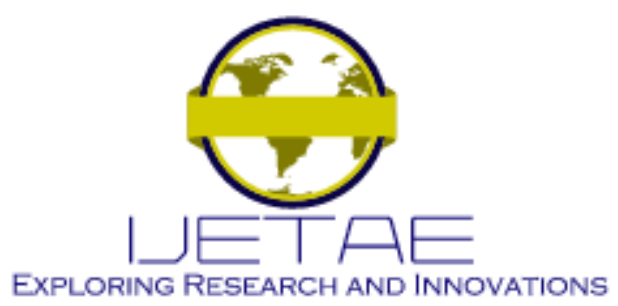

International Journal of Emerging Technology and Advanced Engineering

Website: www.ijetae.com (E-ISSN 2250-2459, Scopus Indexed, ISO 9001:2008 Certified Journal, Volume 11, Issue 10, October 2021)

The web server selects lightweight tomcat, but deploys its server on the cloud server and connects the cloud database with JDBC database connection pool ${ }^{[33]}$. In order to realize the resource sharing of aircraft load spectrum database, Hu etc. used ASP technology and Unified Modeling Language to design the aircraft load spectrum database network system ${ }^{[34]}$.

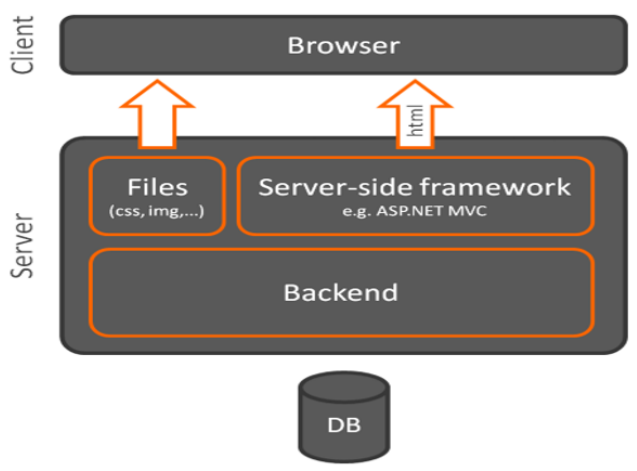

Fig 8 Mode of B / S architecture

\section{E. Database design}

Database design refers to the process of designing the database structure and establishing the database on a specific database management system according to the needs of users. In the process of software development, the design of database plays an extremely important role. An excellent database is conducive to saving resources, improving software running speed and reducing software faults. Generally, the design of the database takes at least $40 \%$ of the development time of the whole project. After the database design is completed, $80 \%$ of the design and development of the project has been completed in mind ${ }^{[35]}$.

The design of load spectrum database can be roughly divided into six stages: demand analysis, conceptual structure design, logical structure design, physical structure design, database implementation and maintenance, and database operation and maintenance, as shown in Figure 9.
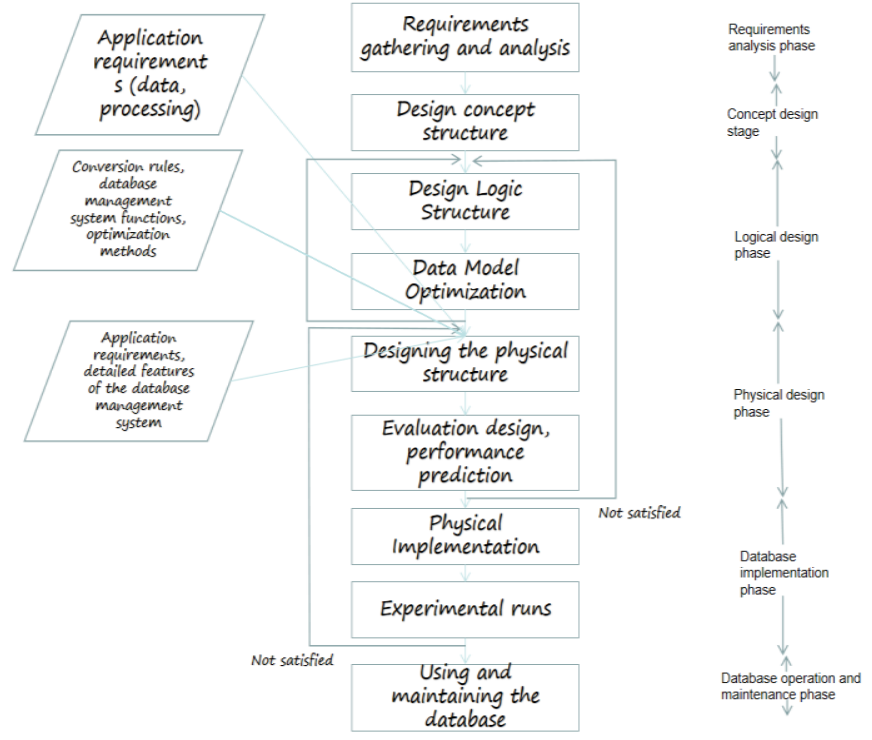

Fig 9 Flow chart of database design

\section{F. Database connection technology}

In the research of load spectrum database, how to connect the database and interact with the database is not only an important problem, but also the focus of database design. Generally, programmers write programs or use reserved interfaces to complete database connection. Common database connection methods include JDBC connection, ADO.Net connection, ODBC connection, database connection pool technology, etc. The following focuses on the common database connection pool technology.

Database connection pool is similar to establishing a "storage pool" for database connections. The Tomcat server puts a certain number of connections in the "connection pool" in advance. When the program needs to establish a connection, it only needs to apply for a connection from the "connection pool", and then put the connection into the "connection pool" after use, so that other programs can apply for it ${ }^{[36]}$. 


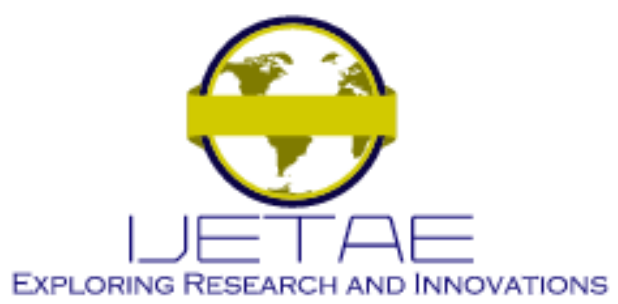

International Journal of Emerging Technology and Advanced Engineering

Website: www.ijetae.com (E-ISSN 2250-2459, Scopus Indexed, ISO 9001:2008 Certified Journal, Volume 11, Issue 10, October 2021)

The database connection pool mechanism can not only improve the speed of database processing connections, but also manage and monitor the number of database connections through the connection pool, so as to provide a basis for system development, testing and performance adjustment ${ }^{[37]}$. The technical principle of database connection pool is shown in Figure 10.

The database connection needs to consider different operating systems, different platforms and different programming languages to comprehensively select the connection mode. The concept of logical data channel proposed by Tian etc. who put forward the physical differences of different data structures ${ }^{[38]}$. The built aircraft load spectrum measured data double buffer visual simulation system opens up two buffers in memory. Buffer $A$ is used to store the measured data obtained from the load spectrum database, and buffer B is used to store the data of buffer A after verification. It greatly ensures the data transmission efficiency and accuracy; Lin etc. used OLEDB for database connection, used ADO database access technology to dynamically add, delete, modify and query the required database, and modified the access database by programming ${ }^{[39]}$. Liu etc. adopted the connection mode based on JDBC database connection pool, and established three mechanisms: database connection mechanism, database management mechanism and database operation mechanism through the connection pool to ensure the connection between the analysis platform and the database, which greatly improves the connection efficiency ${ }^{[40] ;} \mathrm{Xu}$ etc. used the MyBatis framework to manage the data persistence layer and used the objectoriented connector JDBC connection pool to connect to the database $^{[41]}$. Wang etc. used ODBC to establish SQL Server data source in LabVIEW, and then can connect the data source with the lowest ActiveX ${ }^{[42]}$.

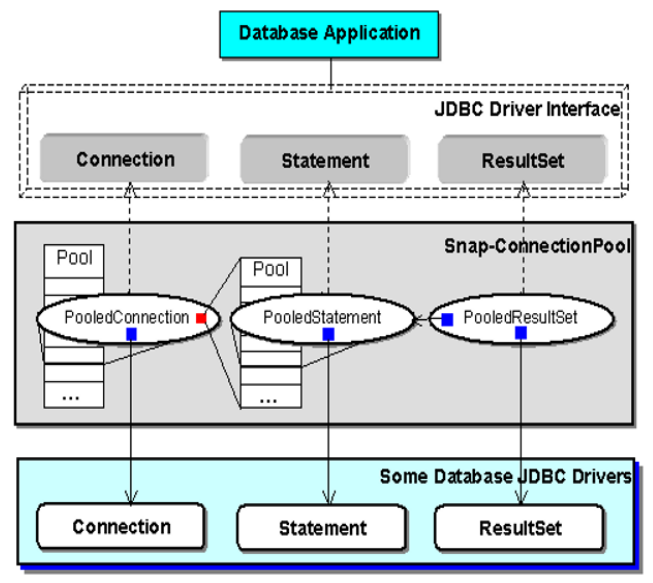

Fig 10 Digram of database connection pooling technology ${ }^{[43]}$

\section{EXPECATION}

With the development of computer technology and database technology, using computer technology to solve engineering problems has become the mainstream trend of scientific research. The efficient computing power of computer can liberate researchers from complex computing. The shared nature of distributed database enables researchers to share experimental data. New energy vehicles are the general trend of the development of vehicles in the future while the major manufacturers building cars one after another. In the process of vehicle development, it is necessary to carry out strength and fatigue tests on the transmission system. Load spectrum is undoubtedly the basis of component reliability design. The amount of data for load spectrum is bound to show an explosive growth trend, and the demand for data management is also increasing. It is an inevitable trend to store and manage load spectrum data with database technology. 


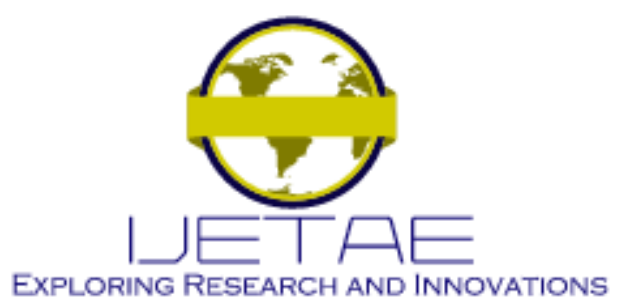

International Journal of Emerging Technology and Advanced Engineering

Website: www.ijetae.com (E-ISSN 2250-2459, Scopus Indexed, ISO 9001:2008 Certified Journal, Volume 11, Issue 10, October 2021)

However, at present, there has little research for the load spectrum database. The application technology is relatively old. How to apply database technology in the field of load fatigue is the main consideration. The research trends are as follows:

(1) Develop a load spectrum database with an engineering thought to improve development efficiency. With the in-depth study of software engineering theory and the continuous development of software technology, software analysis modeling is becoming more and more perfect. The modular design idea is adopted, the modeling software is introduced for development, and the development model suitable for the load spectrum database is selected. In order to meet the needs of users, a load spectrum database with excellent performance is developed in a short time and at a low cost.

(2) The development mode of combining B / S and C / S mode is adopted. It absorbs the respective advantages of $\mathrm{B} / \mathrm{S}$ structure and C / S structure, and integrated them. Not only the powerful data operation and transaction processing capability of $\mathrm{C} / \mathrm{S}$ structure, but also the easy maintenance and expansion of B / S structure, the characteristics of better sharing. The two complement each other, become a practical solution for the scientific management of load spectrum data. For example, Meng etc. chose the B / S system as the main body and the C / $\mathrm{S}$ system in the high-speed interactive part with large amount of data from the actual requirements of the data management system ${ }^{[44]}$.

(3) Database security should be paid attention to.

Database security refers to protecting the database from data leakage, change or damage caused by illegal use because a large number of important and confidential data are stored in the load spectrum database system. In the new network environment, the attack methods against the database are also emerging one after another. The database system has to face more security threats, and the security of the data system is becoming more and more important.

\section{Acknowledgements}

This work was supported by the Key Research and Development Project of Guangdong Province, "High Efficiency, High Precision, Long life and Low noise New energy Vehicle Gearbox Related technology development and application" (No: 2020B090926004).

\section{REFERENCES}

[1] Kong Xiannian, Tang Jinyuan, Siyu Chen, Hu Zehua. Effects of gearbox housing flexibility on dynamic characteristics of gear transmission system[J]. Journal of Vibration and Control,2021,27(17-18):27-42.

[2] Li Yong, Hu Wensheng, Fu Xiaoli, Yang Shufeng, Sun Qiantao, Jin Yuzhe. Current situation and development trend of fatigue life research of gearbox case [J]. Journal of Zhongyuan Institute of Technology, 2016,27 (3): 26-29.

[3] $\mathrm{Hu}$ Weigang. Study on load characteristics and strain field identification method of high-speed train gearbox [D]. Beijing Beijing Jiaotong University, 2019.

[4] Bai Xuewen, Qi Zhihui, Wu Yan, Liu Qian, Bai Wentong. Application of road simulation test technology in the development of new energy vehicles [J]. Vibration and impact, 2019,38 (6): 83-87 + 113.

[5] Wang Shanming. Research on key technologies for compiling load spectrum of commercial vehicle transmission [D]. Taiyuan Taiyuan University of technology, 2018.

[6] Wang Zhenyu, Wang Naixiang, Wang Jixin, Zhang yingshuang. Overview of gear load spectrum compilation method based on mixed distribution [J]. Mining machinery, 2011,39 (4): 11-13.

[7] Xiao Xiaohui, Wu Gongping, Li Libin. Compilation of fatigue load spectrum of tower crane [J]. Journal of applied mechanics, 2003 (4): $86-88+166$

[8] Zhang Yingshuang, Wang Guoqiang, Wang Jixin, Jia Haibo, Zhang Erping. Compilation method of load spectrum of engineering vehicle transmission system [J]. Journal of agricultural engineering, 2011,27 (4): 179-183.

[9] Mou Kaiyu, Wei Ming, Yang Hui, Peng Zhen. Blink artifact removal method based on wavelet singularity detection and threshold denoising [J]. Beijing Biomedical Engineering, 2015,34 (3): 251-255 +283 .

[10] Yan Hengna, Zhao Peiyu, Zhong Guoyang, Yang Tong, Liu Xiujun. Statistical analysis method of Vehicle measured load [J]. China automotive, 2018 (12): 44-48.

[11] Xu Kejun, Wang Yongqi, Xia Yirui, Qin Haiqin. Influence analysis of small amplitude load cycle based on flight parameters on fatigue life calculation [J]. Mechanical strength, 2016,38 (3): 618-625.

[12] Guo Hu, Deng Yaowen, Wu Huimin, Chen Junxiang, Zhu Yongguo. Statistical analysis of vehicle random load spectrum [J]. Automotive science and technology, 2003 (6): 43-45.

[13] Liu Tianqi, Qi Xinxin, Shi Xinhong, Gao Limin, Zhang Tian, Zhang Jianyu. Effect of Loading Frequency Ratio on Multiaxial Asynchronous Fatigue Failure of $30 \mathrm{CrMnSiA}$ Steel[J]. Materials,2021,14(14):23-32.

[14] Xing Guangpeng. Study on applicability of cycle counting method in Aeroengine load spectrum statistics [D]. Nanjing Nanjing University of Aeronautics and Astronautics, 2017.

[15] Rain Flow Matrix.www.researchgate.net.

[16] Li Binchao, Tang Jing, Yin Zhiping. Compilation of engine vibration fatigue load spectrum based on frequency rain flow counting method [J]. Progress of aviation engineering, 2021,12 (1): 24-29. 


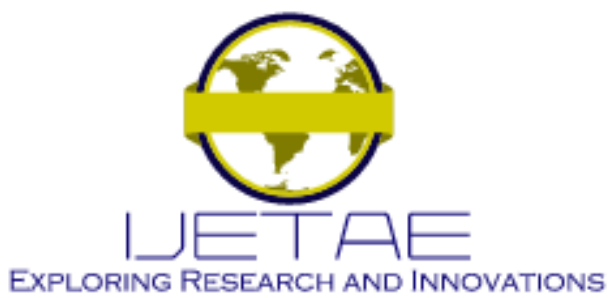

International Journal of Emerging Technology and Advanced Engineering

Website: www.ijetae.com (E-ISSN 2250-2459, Scopus Indexed, ISO 9001:2008 Certified Journal, Volume 11, Issue 10, October 2021)

[17] Zhang Fuze. Study on a new method of compiling aircraft payload [J]. Journal of Aeronautics, 1998, 19 (5): 518-524.

[18] Jiang Dongfang. Recursive algorithm for rain flow counting [J]. Journal of Aeronautics, 2009, 30 (1): 99-103.

[19] Gao Yunfei, Zhang Xiufen, Xue Junfang. Study on gear program load spectrum based on rain flow counting method $[\mathrm{J}]$. Machine tool and hydraulic, 2020,48 (7): 11-15.

[20] S.H.BAEK, S.S.CHO, W.S.JOO, Yan Feng. Fatigue life prediction of freight car bogie end beam based on rain flow cycle counting method [J]. Foreign railway vehicles, 2021,58 (3): 39-44.

[21] Zheng Ruyan, Zhang Kun, Tian Jun. study on fatigue damage assessment method of hull structure based on rain flow counting method [J]. Electromechanical equipment, 2021,38 (3): 112-116.

[22] Ginalis Christine L, Zaki Jeenia, Bedoya Ana Cristina, Nomura Yoko. A-44 Implications of the Heart Rate Variability Power Spectrum on the Relationship between Prenatal Anxiety Exposure and Child Anxiety in 5-Year-Olds[J]. Archives of Clinical Neuropsychology,2021,36(6): 25-30.

[23] Yu Xiaohong, Zhang Laibin, Wang Zhaohui, Duan Lixiang. Equipment life reliability analysis based on new Weibull distribution parameter estimation method [J]. Mechanical strength, 2007 (6): 932-936.

[24] Fu Min, Li Runrun. An improved nonparametric power spectrum estimation method $[\mathrm{J}]$. Journal of Chifeng University (NATURAL SCIENCE EDITION), 2016,32 (9): 17-18.

[25] Chai Chengliang, Li Guoliang, Zhao Tianyu, Luo Yuyu, Yu Minghe. Overview of crowdsourcing database [J]. Journal of computer science, 2020,43 (5): 948-972.

[26] Zhou Fuqiang, Zhang Shuming, Yan Chuliang. Database design of fatigue / fracture properties of typical aircraft materials -- Based on Java applet $[\mathrm{J}]$. Computer engineering and application, 2007 (17): 245-248.

[27] Liu Zuming, Cao Dingguo, Wu Youjin, Zhang Jinhua, Wang Haowei, Luo Jiashu. Research on aircraft structural corrosion control design database [J]. Journal of Aeronautics, 2002 (4): 360-363.

[28] Tian Zhaofeng, Yan Chuliang. A design method of aircraft load spectrum database system based on RIA structure [J]. Acta Aeronautica Sinica, 2009,30 (10): 1889-1894.

[29] Hu Jiyang. Research on the necessity and design method of computer software database development [J]. Computer knowledge and technology, 2020,16 (29): 36-37.
[30] Yan Chuliang, Zhang Shuming, Ye Ge, Gao Zhentong. Median random load spectrum data processing expert system [J]. Journal of Beijing University of Aeronautics and Astronautics, 2000 (4): 428431.

[31] Tian Yong. Aircraft calendar life calculation and evaluation system [D]. Xi'an Northwest University of technology, 2001.

[32] Shen Ruigang, Liu Jianhua, Wu Gongping, Chen Qinghao. Residual life evaluation system of tower crane based on client / server model [J]. Hoisting and transportation machinery, 2003 (5): 21-23.

[33] Liu Hailin. Research and development of network-based multiaxial strain fatigue analysis platform [D]. Dalian Dalian University of technology, 2016.

[34] Hu Yuxuan. Application of ASP and UML modeling technology in aircraft load spectrum database design [D]. China Academy of agricultural mechanization, 2007.

[35] Du Sheng. Design trend of distributed relational database [n]. China informatization weekly, 2021-04-19 (26).

[36] Chang Zuzheng. Working principle and configuration of Tomcat database connection pool $[\mathrm{J}]$. Information and computer (theoretical Edition), 2017 (18): 120-121 + 124 .

[37] Liu Cuixia. Principle and application of Java database connection pool [J]. Wireless Internet technology, 2020,17 (04): 167-168.

[38] Tian Zhaofeng, Yan Chuliang. Design of double buffer visual simulation system for measured data of aircraft load spectrum [J]. Journal of Beijing University of Aeronautics and Astronautics, 2015,41 (3): 431-436.

[39] Lin Xiangyu. Performance research and structural design system development of high-power fracturing vehicle frame [D]. Dalian Dalian University of technology, 2017.

[40] Liu Hailin. Research and development of network-based multiaxial strain fatigue analysis platform [D]. Dalian Dalian University of technology, 2016.

[41] Xu Qiang. Research and development of fatigue life analysis network platform based on damage tolerance [D]. Dalian Dalian University of technology, 2019.

[42] Wang Cheng. Establishment and application of military special vehicle test data management system [D]. Chongqing Chongqing University, 2018.

[43] Infroworld.www.Infoworld.com2021,7,30.

[44] Meng Qingrui, Tian Zhaofeng, Zhang Shuming, Yan Chuliang. Load spectrum data management system of agricultural machinery under mixed mode[j] Journal of agricultural machinery. 2009 (4): 360-363. 